\title{
SECOND-ORDER $n$-POINT EIGENVALUE PROBLEMS ON TIME SCALES
}

DOUGLAS R. ANDERSON AND RUYUN MA

Received 10 December 2004; Revised 3 November 2005; Accepted 6 November 2005

We discuss conditions for the existence of at least one positive solution to a nonlinear second-order Sturm-Liouville-type multipoint eigenvalue problem on time scales. The results extend previous work on both the continuous case and more general time scales, and are based on the Guo-Krasnosel'skii fixed point theorem.

Copyright (C) 2006 D. R. Anderson and R. Ma. This is an open access article distributed under the Creative Commons Attribution License, which permits unrestricted use, distribution, and reproduction in any medium, provided the original work is properly cited.

\section{Introduction}

We are interested in the second-order multipoint time-scale eigenvalue problem

$$
\begin{gathered}
\left(p y^{\nabla}\right)^{\Delta}(t)-q(t) y(t)+\lambda h(t) f(y)=0, \quad t_{1}<t<t_{n}, \\
\alpha y\left(t_{1}\right)-\beta p\left(t_{1}\right) y^{\nabla}\left(t_{1}\right)=\sum_{i=2}^{n-1} a_{i} y\left(t_{i}\right), \quad \gamma y\left(t_{n}\right)+\delta p\left(t_{n}\right) y^{\nabla}\left(t_{n}\right)=\sum_{i=2}^{n-1} b_{i} y\left(t_{i}\right),
\end{gathered}
$$

where

$$
p, q:\left[t_{1}, t_{n}\right] \longrightarrow(0, \infty), \quad p \in C^{\Delta}\left[t_{1}, t_{n}\right), q \in C\left[t_{1}, t_{n}\right]
$$

the points $t_{i} \in \mathbb{T}_{\kappa}^{\kappa}$ for $i \in\{1,2, \ldots, n\}$ with $t_{1}<t_{2}<\cdots<t_{n}$;

$$
\alpha, \beta, \gamma, \delta \in[0, \infty), \quad \alpha \gamma+\alpha \delta+\beta \gamma>0, \quad a_{i}, b_{i} \in[0, \infty), \quad i \in\{2, \ldots, n-1\} .
$$

The continuous function $f:[0, \infty) \rightarrow[0, \infty)$ is such that the following exist:

$$
f_{0}:=\lim _{y \rightarrow 0^{+}} \frac{f(y)}{y}, \quad f_{\infty}:=\lim _{y \rightarrow \infty} \frac{f(y)}{y}
$$

Hindawi Publishing Corporation Advances in Difference Equations Volume 2006, Article ID 59572, Pages 1-17

DOI 10.1155/ADE/2006/59572 
and the right-dense continuous function $h:\left[t_{1}, t_{n}\right] \rightarrow[0, \infty)$ satisfies some suitable conditions to be developed. Problem (1.1), (1.2) is a generalization to time scales of the problem when $\mathbb{T}$ is restricted to $\mathbb{R}$ on the unit interval in Ma and Thompson [19], and extends the type of time-scale boundary value problem found in Anderson [2], Atici and Guseinov [6], Kaufmann [15], Kaufmann and Raffoul [16], and Sun and Li [21, 22]. Other related three-point problems on time scales include Anderson and Avery [4], Anderson et al. [5], Peterson et al. [20], and a singular problem in DaCunha et al. [12]. Some of the work on multipoint time-scale problems includes Anderson [1, 3] and Kong and Kong [17], and a recent singular multipoint problem in Bohner and Luo [8]. For more general information concerning dynamic equations on time scales, introduced by Aulbach and Hilger [7] and Hilger [14], see the excellent text by Bohner and Peterson [9] and their edited text [10].

\section{Time-scale primer}

Any arbitrary nonempty closed subset of the reals $\mathbb{R}$ can serve as a time-scale $\mathbb{T}$; see $[9$, $10]$. For $t \in \mathbb{T}$ define the forward jump operator $\sigma: \mathbb{T} \rightarrow \mathbb{T}$ by $\sigma(t)=\inf \{s \in \mathbb{T}: s>t\}$, and the backward jump operator $\rho: \mathbb{T} \rightarrow \mathbb{T}$ by $\rho(t)=\sup \{s \in \mathbb{T}: s<t\}$. The graininess operators $\mu_{\sigma}, \mu_{\rho}: \mathbb{T} \rightarrow[0, \infty)$ are defined by $\mu_{\sigma}(t)=\sigma(t)-t$ and $\mu_{\rho}(t)=\rho(t)-t$.

A function $f: \mathbb{T} \rightarrow \mathbb{R}$ is right-dense continuous (rd-continuous) provided it is continuous at all right-dense points of $\mathbb{T}$ and its left-sided limit exists (is finite) at leftdense points of $\mathbb{T}$. The set of all right-dense continuous functions on $\mathbb{T}$ is denoted by $C_{r d}=C_{r d}(\mathbb{T})=C_{r d}(\mathbb{T}, \mathbb{R})$.

Define the set $\mathbb{T}_{\kappa}$ by $\mathbb{T}_{\kappa}=\mathbb{T}-\{m\}$ if $\mathbb{T}$ has a right scattered minimum $m$ and $\mathbb{T}_{\kappa}=\mathbb{T}$ otherwise. In a similar vein, $\mathbb{T}^{\kappa}=\mathbb{T}-\{M\}$ if $\mathbb{T}$ has a left scattered maximum $M$ and $\mathbb{T}^{\kappa}=\mathbb{T}$ otherwise. We take $\mathbb{T}_{\kappa}^{\kappa}=\mathbb{T}_{\kappa} \cap \mathbb{T}^{\kappa}$.

Definition 2.1 (delta derivative). Assume $f: \mathbb{T} \rightarrow \mathbb{R}$ is a function and let $t \in \mathbb{T}^{\kappa}$. Define $f^{\Delta}(t)$ to be the number (provided it exists) with the property that given any $\epsilon>0$, there is a neighborhood $U \subset \mathbb{T}$ of $t$ such that

$$
\left|[f(\sigma(t))-f(s)]-f^{\Delta}(t)[\sigma(t)-s]\right| \leq \epsilon|\sigma(t)-s| \quad \forall s \in U
$$

The function $f^{\Delta}(t)$ is the delta derivative of $f$ at $t$.

Definition 2.2 (nabla derivative). For $f: \mathbb{T} \rightarrow \mathbb{R}$ and $t \in \mathbb{T}_{\kappa}$, define $f^{\nabla}(t)$ to be the number (provided it exists) with the property that given any $\epsilon>0$, there is a neighborhood $U$ of $t$ such that

$$
\left|f(\rho(t))-f(s)-f^{\nabla}(t)[\rho(t)-s]\right| \leq \epsilon|\rho(t)-s| \quad \forall s \in U
$$

The function $f^{\nabla}(t)$ is the nabla derivative of $f$ at $t$.

In the case $\mathbb{T}=\mathbb{R}, f^{\Delta}(t)=f^{\prime}(t)=f^{\nabla}(t)$. When $\mathbb{T}=\mathbb{Z}, f^{\Delta}(t)=f(t+1)-f(t)$ and $f^{\nabla}(t)=f(t)-f(t-1)$. 
Definition 2.3 (delta integral). Let $f: \mathbb{T} \rightarrow \mathbb{R}$ be a function, and let $a, b \in \mathbb{T}$. If there exists a function $F: \mathbb{T} \rightarrow \mathbb{R}$ such that $F^{\Delta}(t)=f(t)$ for all $t \in \mathbb{T}^{\kappa}$, then $F$ is a delta antiderivative of $f$. In this case the integral is given by the formula

$$
\int_{a}^{b} f(t) \Delta t=F(b)-F(a) \quad \text { for } a, b \in \mathbb{T} .
$$

All right-dense continuous functions are delta integrable; see [9, Theorem 1.74].

\section{Linear preliminaries}

We first construct Green's function for the second-order boundary value problem

$$
\begin{gathered}
\left(p y^{\nabla}\right)^{\Delta}(t)-q(t) y(t)+u(t)=0, \quad t_{1}<t<t_{n}, \\
\alpha y\left(t_{1}\right)-\beta p\left(t_{1}\right) y^{\nabla}\left(t_{1}\right)=0, \quad \gamma y\left(t_{n}\right)+\delta p\left(t_{n}\right) y^{\nabla}\left(t_{n}\right)=0,
\end{gathered}
$$

where $\alpha, \beta, \gamma, \delta$ are real numbers such that $|\alpha|+|\beta| \neq 0,|\gamma|+|\delta| \neq 0$. The techniques here are similar to those found in $[6,19]$.

Denote by $\phi$ and $\psi$ the solutions of the corresponding homogeneous equation

$$
\left(p y^{\nabla}\right)^{\Delta}(t)-q(t) y(t)=0, \quad t \in\left[t_{1}, t_{n}\right)
$$

under the initial conditions

$$
\begin{array}{ll}
\psi\left(t_{1}\right)=\beta, & p\left(t_{1}\right) \psi^{\nabla}\left(t_{1}\right)=\alpha, \\
\phi\left(t_{n}\right)=\delta, & p\left(t_{n}\right) \phi^{\nabla}\left(t_{n}\right)=-\gamma
\end{array}
$$

so that $\psi$ and $\phi$ satisfy the first and second boundary conditions in (3.2), respectively. Set

$$
d=-W_{t}(\psi, \phi)=p(t) \psi^{\nabla}(t) \phi(t)-\psi(t) p(t) \phi^{\nabla}(t) .
$$

Since the Wronskian of any two solutions is independent of $t$, evaluating at $t=t_{1}, t=t_{n}$, and using the boundary conditions (3.4), (3.5) yields

$$
d=\alpha \phi\left(t_{1}\right)-\beta p\left(t_{1}\right) \phi^{\nabla}\left(t_{1}\right)=\gamma \psi\left(t_{n}\right)+\delta p\left(t_{n}\right) \psi^{\nabla}\left(t_{n}\right)
$$

In addition $d \neq 0$ if and only if the homogeneous equation (3.3) has only the trivial solution satisfying the boundary conditions (3.2). For the proof of the following theorem, see $[6$, Theorem 4.2$]$.

Lemma 3.1. Assume (1.3) and (1.4). If $d \neq 0$, then the nonhomogeneous boundary value problem (3.1)-(3.2) has a unique solution y for which the formula

$$
y(t)=\int_{t_{1}}^{t_{n}} G(t, s) u(s) \Delta s, \quad t \in\left[\rho\left(t_{1}\right), t_{n}\right]
$$


4 Second-order $n$-point eigenvalue problems on time scales

holds, where the function $G(t, s)$ is given by

$$
G(t, s)=\frac{1}{d} \begin{cases}\psi(t) \phi(s), & \rho\left(t_{1}\right) \leq t \leq s \leq t_{n} \\ \psi(s) \phi(t), & \rho\left(t_{1}\right) \leq s \leq t \leq t_{n}\end{cases}
$$

and $G(t, s)$ is Green's function of the boundary value problem (3.1)-(3.2). Furthermore Green's function is symmetric, that is, $G(t, s)=G(s, t)$ for $t, s \in\left[\rho\left(t_{1}\right), t_{n}\right]$.

Lemma 3.2. Assume (1.3) and (1.4). Then the functions $\psi$ and $\phi$ satisfy

$$
\begin{gathered}
\psi(t) \geq 0, \quad t \in\left[\rho\left(t_{1}\right), t_{n}\right], \quad \psi(t)>0, \quad t \in\left(\rho\left(t_{1}\right), t_{n}\right], \\
p(t) \psi^{\nabla}(t) \geq 0, \quad t \in\left[\rho\left(t_{1}\right), t_{n}\right], \quad \phi(t) \geq 0, \quad t \in\left[\rho\left(t_{1}\right), t_{n}\right], \\
\phi(t)>0, \quad t \in\left[\rho\left(t_{1}\right), t_{n}\right), \quad p(t) \phi^{\nabla}(t) \leq 0, \quad t \in\left[\rho\left(t_{1}\right), t_{n}\right] .
\end{gathered}
$$

Proof. The proof is very similar to the proof of [6, Lemma 5.1] and is omitted.

Set

$$
D:=\left|\begin{array}{cc}
-\sum_{i=2}^{n-1} a_{i} \psi\left(t_{i}\right) & d-\sum_{i=2}^{n-1} a_{i} \phi\left(t_{i}\right) \\
d-\sum_{i=2}^{n-1} b_{i} \psi\left(t_{i}\right) & -\sum_{i=2}^{n-1} b_{i} \phi\left(t_{i}\right)
\end{array}\right| .
$$

Lemma 3.3. Assume (1.3) and (1.4). If $D \neq 0$ and $u \in C_{r d}\left[t_{1}, t_{n}\right]$, then the nonhomogeneous dynamic equation (3.1) with boundary conditions (1.2) has a unique solution $y$ for which the formula

$$
y(t)=\int_{t_{1}}^{t_{n}} G(t, s) u(s) \Delta s+A(u) \psi(t)+B(u) \phi(t), \quad t \in\left[\rho\left(t_{1}\right), t_{n}\right]
$$

holds, where the function $G(t, s)$ is Green's function (3.9) of the boundary value problem (3.1)-(3.2) and the functionals $A$ and $B$ are defined by

$$
\begin{aligned}
& A(u):=\frac{1}{D}\left|\begin{array}{ll}
\sum_{i=2}^{n-1} a_{i} \int_{t_{1}}^{t_{n}} G\left(t_{i}, s\right) u(s) \Delta s & d-\sum_{i=2}^{n-1} a_{i} \phi\left(t_{i}\right) \\
\sum_{i=2}^{n-1} b_{i} \int_{t_{1}}^{t_{n}} G\left(t_{i}, s\right) u(s) \Delta s & -\sum_{i=2}^{n-1} b_{i} \phi\left(t_{i}\right)
\end{array}\right|, \\
& B(u):=\frac{1}{D}\left|\begin{array}{cc}
-\sum_{i=2}^{n-1} a_{i} \psi\left(t_{i}\right) & \sum_{i=2}^{n-1} a_{i} \int_{t_{1}}^{t_{n}} G\left(t_{i}, s\right) u(s) \Delta s \\
d-\sum_{i=2}^{n-1} b_{i} \psi\left(t_{i}\right) & \sum_{i=2}^{n-1} b_{i} \int_{t_{1}}^{t_{n}} G\left(t_{i}, s\right) u(s) \Delta s
\end{array}\right| .
\end{aligned}
$$


Proof. It can be verified that for a solution $y$ of the nonhomogeneous equation (3.1) under the nonhomogeneous boundary conditions (1.2), the formula (3.12) holds, where $G(t, s)$ is given by (3.9). We thus show that the function $y$ given in (3.12) is a solution of (3.1) with conditions (1.2) only if $A$ and $B$ are given by (3.13) and (3.14), respectively. If $y$ as in (3.12) is a solution of (3.1), (1.2), then

$$
y(t)=\frac{1}{d} \int_{t_{1}}^{t} \phi(t) \psi(s) u(s) \Delta s+\frac{1}{d} \int_{t}^{t_{n}} \psi(t) \phi(s) u(s) \Delta s+A \psi(t)+B \phi(t)
$$

for some constants $A$ and $B$. Taking the nabla derivative and multiplying by $p$ yields

$$
p y^{\nabla}=\frac{p \phi^{\nabla}}{d} \int_{t_{1}}^{t} \psi(s) u(s) \Delta s+\frac{p \psi^{\nabla}}{d} \int_{t}^{t_{n}} \phi(s) u(s) \Delta s+A p \psi^{\nabla}+B p \phi^{\nabla}
$$

the delta derivative of this expression is

$$
\begin{aligned}
\left(p y^{\nabla}\right)^{\Delta}= & \left(\frac{p \phi^{\nabla}}{d}\right)^{\Delta} \int_{t_{1}}^{\sigma(t)} \psi(s) u(s) \Delta s+\frac{p \phi^{\nabla}}{d} \psi(t) u(t)+A\left(p \psi^{\nabla}\right)^{\Delta}+B\left(p \phi^{\nabla}\right)^{\Delta} \\
& +\left(\frac{p \psi^{\nabla}}{d}\right)^{\Delta} \int_{\sigma(t)}^{t_{n}} \phi(s) u(s) \Delta s-\frac{p \psi^{\nabla}}{d} \phi(t) u(t) .
\end{aligned}
$$

Using [9, Theorem 1.75], and the fact that $\psi$ and $\phi$ are solutions to (3.3), we obtain

$$
\begin{aligned}
\left(p y^{\nabla}\right)^{\Delta}(t)= & \frac{q(t)}{d} \int_{t_{1}}^{t} \phi(t) \psi(s) u(s) \Delta s+\frac{q(t)}{d} \phi(t) \mu_{\sigma}(t) \psi(t) u(t)+\frac{u(t)}{d} p(t) \phi^{\nabla}(t) \psi(t) \\
& +\frac{q(t)}{d} \int_{t}^{t_{n}} \psi(t) \phi(s) u(s) \Delta s-\frac{q(t)}{d} \psi(t) \mu_{\sigma}(t) \phi(t) u(t) \\
& -\frac{u(t)}{d} p(t) \psi^{\nabla}(t) \phi(t)+q(t)(A \psi(t)+b \phi(t)) .
\end{aligned}
$$

Recall that $d$ is in terms of the Wronskian of $\psi$ and $\phi$ in (3.6); it follows that

$$
\left(p y^{\nabla}\right)^{\Delta}(t)=q(t) y(t)-u(t)
$$

Now

$$
\begin{gathered}
y\left(t_{1}\right)=\frac{\psi\left(t_{1}\right)}{d} \int_{t_{1}}^{t_{n}} \phi(s) u(s) \Delta s+A \psi\left(t_{1}\right)+B \phi\left(t_{1}\right), \\
p\left(t_{1}\right) y^{\nabla}\left(t_{1}\right)=\frac{p\left(t_{1}\right) \psi^{\nabla}\left(t_{1}\right)}{d} \int_{t_{1}}^{t_{n}} \phi(s) u(s) \Delta s+A p\left(t_{1}\right) \psi^{\nabla}\left(t_{1}\right)+B p\left(t_{1}\right) \phi^{\nabla}\left(t_{1}\right) ;
\end{gathered}
$$


6 Second-order $n$-point eigenvalue problems on time scales

multiply the first line by $\alpha$ and the second by $-\beta$, and use (1.2) and (3.4) to see that

$$
B\left[\alpha \phi\left(t_{1}\right)-\beta p\left(t_{1}\right) \phi^{\nabla}\left(t_{1}\right)\right]=\sum_{i=2}^{n-1} a_{i}\left(\int_{t_{1}}^{t_{n}} G\left(t_{i}, s\right) u(s) \Delta s+A \psi\left(t_{i}\right)+B \phi\left(t_{i}\right)\right) .
$$

At the other end,

$$
\begin{gathered}
y\left(t_{n}\right)=\frac{\phi\left(t_{n}\right)}{d} \int_{t_{1}}^{t_{n}} \psi(s) u(s) \Delta s+A \psi\left(t_{n}\right)+B \phi\left(t_{n}\right), \\
p\left(t_{n}\right) y^{\nabla}\left(t_{n}\right)=\frac{p\left(t_{n}\right) \phi^{\nabla}\left(t_{n}\right)}{d} \int_{t_{1}}^{t_{n}} \psi(s) u(s) \Delta s+A p\left(t_{n}\right) \psi^{\nabla}\left(t_{n}\right)+B p\left(t_{n}\right) \phi^{\nabla}\left(t_{n}\right) ;
\end{gathered}
$$

consequently

$$
A\left[\gamma \psi\left(t_{n}\right)+\delta p\left(t_{n}\right) \psi^{\nabla}\left(t_{n}\right)\right]=\sum_{i=2}^{n-1} b_{i}\left(\int_{t_{1}}^{t_{n}} G\left(t_{i}, s\right) u(s) \Delta s+A \psi\left(t_{i}\right)+B \phi\left(t_{i}\right)\right) .
$$

Combining (3.21) and (3.23) and using (3.6), we arrive at the system of equations

$$
\begin{gathered}
-A \sum_{i=2}^{n-1} a_{i} \psi\left(t_{i}\right)+B\left[\alpha \phi\left(t_{1}\right)-\beta p\left(t_{1}\right) \phi^{\nabla}\left(t_{1}\right)-\sum_{i=2}^{n-1} a_{i} \phi\left(t_{i}\right)\right]=\sum_{i=2}^{n-1} a_{i} \int_{t_{1}}^{t_{n}} G\left(t_{i}, s\right) u(s) \Delta s, \\
A\left[\gamma \psi\left(t_{n}\right)+\delta p\left(t_{n}\right) \psi^{\nabla}\left(t_{n}\right)-\sum_{i=2}^{n-1} b_{i} \psi\left(t_{i}\right)\right]-B \sum_{i=2}^{n-1} b_{i} \phi\left(t_{i}\right)=\sum_{i=2}^{n-1} b_{i} \int_{t_{1}}^{t_{n}} G\left(t_{i}, s\right) u(s) \Delta s .
\end{gathered}
$$

Again using (3.6) at both $t_{1}$ and $t_{n}$, we verify (3.13) and (3.14).

LEMma 3.4. Let (1.3) and (1.4) hold, and assume

$$
D<0, \quad d-\sum_{i=2}^{n-1} a_{i} \phi\left(t_{i}\right)>0, \quad d-\sum_{i=2}^{n-1} b_{i} \psi\left(t_{i}\right)>0
$$

for $D$ and $d$ given in (3.11) and (3.6), respectively. If $u \in C_{r d}\left[t_{1}, t_{n}\right]$ with $u \geq 0$, the unique solution $y$ as in (3.12) of the problem (3.1), (1.2) satisfies $y(t) \geq 0$ for $t \in\left[t_{1}, t_{n}\right]$.

Proof. From the previous lemmas and assumptions we know that Green's function (3.9) satisfies $G(t, s) \geq 0$ on $\left[\rho\left(t_{1}\right), t_{n}\right] \times\left[\rho\left(t_{1}\right), t_{n}\right]$. Hypotheses (1.3), (1.4), and (3.25) applied to (3.13) and (3.14) imply that $A(u), B(u) \geq 0$.

Suppose (3.25) does not hold. For example, let $n=3, p(t) \equiv 1=\alpha=\gamma, q(t) \equiv 0=\beta=$ $\delta=a_{2}$, and $t_{1}=0$. Then (3.1), (1.2) becomes

$$
y^{\nabla \Delta}(t)+u(t)=0, \quad t_{1}<t<t_{3}, \quad y\left(t_{1}\right)=0, \quad y\left(t_{3}\right)=b_{2} y\left(t_{2}\right)
$$


Note that $\psi(t)=t, d=t_{3}$, and $D=t_{3}\left(b_{2} t_{2}-t_{3}\right)$. If $D>0$, then $b_{2} t_{2}>t_{3}$, and there is no positive solution; see [15, Lemma 4].

Lemma 3.5. Let (1.3), (1.4), and (3.25) hold, and fix

$$
\xi_{1}, \xi_{2} \in \mathbb{T}_{\kappa}^{\kappa}, \quad \rho\left(t_{1}\right)<\xi_{1}<\xi_{2}<t_{n}
$$

If $u \in C_{r d}\left[t_{1}, t_{n}\right]$ with $u \geq 0$, the unique solution $y$ as in (3.12) of the time-scale boundary value problem (3.1), (1.2) satisfies

$$
\min _{t \in\left[\xi_{1}, \xi_{2}\right]} y(t) \geq \Gamma\|y\|, \quad\|y\|:=\max _{t \in\left[\rho\left(t_{1}\right), t_{n}\right]} y(t)
$$

where

$$
\Gamma:=\min \left\{\frac{\phi\left(\xi_{2}\right)}{\phi\left(\rho\left(t_{1}\right)\right)}, \frac{\psi\left(\xi_{1}\right)}{\psi\left(t_{n}\right)}\right\} \in(0,1) .
$$

Proof. From (1.3), (3.9), and Lemma 3.2,

$$
0 \leq G(t, s) \leq G(s, s), \quad t \in\left[\rho\left(t_{1}\right), t_{n}\right],
$$

so that

$$
y(t) \leq \int_{t_{1}}^{t_{n}} G(s, s) u(s) \Delta s+A(u) \psi\left(t_{n}\right)+B(u) \phi\left(\rho\left(t_{1}\right)\right) \quad \forall t \in\left[\rho\left(t_{1}\right), t_{n}\right] .
$$

For $t \in\left[\xi_{1}, \xi_{2}\right]$, Green's function (3.9) satisfies

$$
\frac{G(t, s)}{G(s, s)}=\left\{\begin{array}{ll}
\frac{\phi(t)}{\phi(s)}: & \rho\left(t_{1}\right) \leq s \leq t \leq t_{n} \\
\frac{\psi(t)}{\psi(s)}: & \rho\left(t_{1}\right) \leq t \leq s \leq t_{n}
\end{array} \geq\left\{\begin{array}{ll}
\frac{\phi\left(\xi_{2}\right)}{\phi\left(\rho\left(t_{1}\right)\right)}: & \rho\left(t_{1}\right) \leq s \leq t \leq t_{n} \\
\frac{\psi\left(\xi_{1}\right)}{\psi\left(t_{n}\right)}: & \rho\left(t_{1}\right) \leq t \leq s \leq t_{n}
\end{array} \geq \Gamma\right.\right.
$$

for $\Gamma$ as in (3.29), and

$$
\begin{aligned}
y(t) & =\int_{t_{1}}^{t_{n}} \frac{G(t, s)}{G(s, s)} G(s, s) u(s) \Delta s+A(u) \psi(t)+B(u) \phi(t) \\
& \geq \int_{t_{1}}^{t_{n}} \Gamma G(s, s) u(s) \Delta s+A(u) \psi\left(\xi_{1}\right)+B(u) \phi\left(\xi_{2}\right) \\
& \geq \Gamma\left(\int_{t_{1}}^{t_{n}} G(s, s) u(s) \Delta s+A(u) \psi\left(t_{n}\right)+B(u) \phi\left(\rho\left(t_{1}\right)\right)\right) \geq \Gamma\|y\| .
\end{aligned}
$$

\section{Eigenvalue intervals}

To establish eigenvalue intervals we will employ the following fixed point theorem due to Krasnosel'skii [18]; for more on the establishment of eigenvalue intervals for time-scale boundary value problems, see, for example, Chyan and Henderson [11] and Davis et al. [13]. 
8 Second-order $n$-point eigenvalue problems on time scales

Theorem 4.1. Let $E$ be a Banach space, $P \subseteq E$ a cone, and suppose that $\Omega_{1}, \Omega_{2}$ are bounded open balls of E centered at the origin with $\bar{\Omega}_{1} \subset \Omega_{2}$. Suppose further that $L: P \cap\left(\bar{\Omega}_{2} \backslash \Omega_{1}\right) \rightarrow$ $P$ is a completely continuous operator such that either

(i) $\|L y\| \leq\|y\|, y \in P \cap \partial \Omega_{1}$ and $\|L y\| \geq\|y\|, y \in P \cap \partial \Omega_{2}$, or

(ii) $\|L y\| \geq\|y\|, y \in P \cap \partial \Omega_{1}$ and $\|L y\| \leq\|y\|, y \in P \cap \partial \Omega_{2}$

holds. Then $L$ has a fixed point in $P \cap\left(\bar{\Omega}_{2} \backslash \Omega_{1}\right)$.

Assume that the right-dense continuous function $h$ satisfies

$$
h:\left[t_{1}, t_{n}\right] \longrightarrow[0, \infty), \quad \exists t_{*} \in\left(\sigma\left(t_{1}\right), \rho\left(t_{n}\right)\right) \ni h\left(t_{*}\right)>0 .
$$

Then there exist $\xi_{1}, \xi_{2}$ as in Lemma 3.5 such that

$$
\xi_{1}<t_{*}<\xi_{2}, \quad \int_{\xi_{1}}^{\xi_{2}} G(t, s) h(s) \Delta s>0, \quad t \in\left(\rho\left(t_{1}\right), t_{n}\right)
$$

In the following, let $\Gamma$ be the constant defined in (3.29) with respect to such constants $\xi_{1}, \xi_{2}$. Let $\tau \in\left[\rho\left(t_{1}\right), t_{n}\right]$ be determined by

$$
\int_{\xi_{1}}^{\xi_{2}} G(\tau, s) h(s) \Delta s=\max _{\rho\left(t_{1}\right) \leq t \leq t_{n}} \int_{\xi_{1}}^{\xi_{2}} G(t, s) h(s) \Delta s>0
$$

For $G(t, s)$ in (3.9) and $A, B$ as in (3.13), (3.14), respectively, define the constant

$$
K:=\int_{t_{1}}^{t_{n}} G(s, s) h(s) \Delta s+A(h) \psi\left(t_{n}\right)+B(h) \phi\left(\rho\left(t_{1}\right)\right) .
$$

Let $\mathscr{B}$ denote the Banach space $C\left[\rho\left(t_{1}\right), t_{n}\right]$ with the norm $\|y\|=\sup _{t \in\left[\rho\left(t_{1}\right), t_{n}\right]}|y(t)|$. Define the cone $\mathscr{P} \subset \mathscr{B}$ by

$$
\mathscr{P}=\left\{y \in \mathscr{B}: y(t) \geq 0 \text { on }\left[\rho\left(t_{1}\right), t_{n}\right], y(t) \geq \Gamma\|y\| \text { on }\left[\xi_{1}, \xi_{2}\right]\right\}
$$

where $\Gamma$ is given in (3.29). Since $y$ is a solution of (1.1), (1.2) if and only if

$$
y(t)=\lambda\left(\int_{t_{1}}^{t_{n}} G(t, s) h(s) f(y(s)) \Delta s+A(h f(y)) \psi(t)+B(h f(y)) \phi(t)\right), \quad t \in\left[\rho\left(t_{1}\right), t_{n}\right],
$$


define for $y \in \mathscr{P}$ the operator $T: \mathscr{P} \rightarrow \mathscr{B}$ by

$$
(T y)(t):=\lambda\left(\int_{t_{1}}^{t_{n}} G(t, s) h(s) f(y(s)) \Delta s+A(h f(y)) \psi(t)+B(h f(y)) \phi(t)\right) .
$$

We seek a fixed point of $T$ in $\mathscr{P}$ by establishing the hypotheses of Theorem 4.1.

Theorem 4.2. Suppose (1.3), (1.4), (3.25), (4.1), and (4.3) hold. Then for each $\lambda$ satisfying

$$
\frac{1}{f_{\infty} \Gamma \int_{\xi_{1}}^{\xi_{2}} G(\tau, s) h(s) \Delta s}<\lambda<\frac{1}{f_{0} K}
$$

there exists at least one positive solution of (1.1), (1.2) in $\mathscr{P}$.

Proof. Let $\xi_{1}, \xi_{2}$ be as in Lemma 3.5, let $\tau$ be as in (4.3), let $K$ be as in (4.4), let $\lambda$ be as in (4.8), and let $\epsilon>0$ be such that

$$
\frac{1}{\left(f_{\infty}-\epsilon\right) \Gamma \int_{\xi_{1}}^{\xi_{2}} G(\tau, s) h(s) \Delta s} \leq \lambda \leq \frac{1}{\left(f_{0}+\epsilon\right) K}
$$

Consider the integral operator $T$ in (4.7). If $y \in \mathscr{P}$, then by (3.30) we have

$$
\begin{aligned}
(T y)(t) & =\lambda\left(\int_{t_{1}}^{t_{n}} G(t, s) h(s) f(y(s)) \Delta s+A(h f(y)) \psi(t)+B(h f(y)) \phi(t)\right) \\
& \leq \lambda\left(\int_{t_{1}}^{t_{n}} G(s, s) h(s) f(y(s)) \Delta s+A(h f(y)) \psi\left(t_{n}\right)+B(h f(y)) \phi\left(\rho\left(t_{1}\right)\right)\right),
\end{aligned}
$$

so that for $t \in\left[\xi_{1}, \xi_{2}\right]$,

$$
\begin{aligned}
(T y)(t) & =\lambda\left(\int_{t_{1}}^{t_{n}} G(t, s) h(s) f(y(s)) \Delta s+A(h f(y)) \psi(t)+B(h f(y)) \phi(t)\right) \\
& \geq \lambda\left(\int_{t_{1}}^{t_{n}} \frac{G(t, s)}{G(s, s)} G(s, s) h(s) f(y(s)) \Delta s+A(h f(y)) \psi\left(\xi_{1}\right)+B(h f(y)) \phi\left(\xi_{2}\right)\right) \\
& \geq \lambda \Gamma\left(\int_{t_{1}}^{t_{n}} G(s, s) h(s) f(y(s)) \Delta s+A(h f(y)) \psi\left(t_{n}\right)+B(h f(y)) \phi\left(\rho\left(t_{1}\right)\right)\right) \geq \Gamma\|T y\| .
\end{aligned}
$$

Therefore $T: \mathscr{P} \rightarrow \mathscr{P}$. Moreover, $T$ is completely continuous by a typical application of the Ascoli-Arzela theorem. 
10 Second-order $n$-point eigenvalue problems on time scales

Now consider $f_{0}$. There exists an $R_{1}>0$ such that $f(y) \leq\left(f_{0}+\epsilon\right) y$ for $0<y \leq R_{1}$ by the definition of $f_{0}$. Pick $y \in \mathscr{P}$ with $\|y\|=R_{1}$. From (3.13) and (3.14),

$$
|A(h f(y))| \leq A(h)\|f(y)\|, \quad|B(h f(y))| \leq B(h)\|f(y)\| .
$$

Using (3.30), we have

$$
\begin{aligned}
(T y)(t) & =\lambda\left(\int_{t_{1}}^{t_{n}} G(t, s) h(s) f(y(s)) \Delta s+A(h f(y)) \psi(t)+B(h f(y)) \phi(t)\right) \\
& \leq \lambda\|f(y)\|\left(\int_{t_{1}}^{t_{n}} G(s, s) h(s) \Delta s+A(h) \psi\left(t_{n}\right)+B(h) \phi\left(\rho\left(t_{1}\right)\right)\right) \\
& \leq \lambda\left(f_{0}+\epsilon\right)\|y\| K \leq\|y\|
\end{aligned}
$$

from the right-hand side of (4.9). As a result, $\|T y\| \leq\|y\|$. Thus, take

$$
\Omega_{1}:=\left\{y \in \mathscr{B}:\|y\|<R_{1}\right\}
$$

so that $\|T y\| \leq\|y\|$ for $y \in \mathscr{P} \cap \partial \Omega_{1}$.

Next consider $f_{\infty}$. Again by definition, there exists an $R_{2}^{\prime}>R_{1}$ such that $f(y) \geq\left(f_{\infty}-\right.$ $\epsilon) y$ for $y \geq R_{2}^{\prime}$; take $R_{2}=\max \left\{2 R_{1}, R_{2}^{\prime} / \Gamma\right\}$. If $y \in \mathscr{P}$ with $\|y\|=R_{2}$, then for $s \in\left[\xi_{1}, \xi_{2}\right]$ we have

$$
y(s) \geq \Gamma\|y\|=\Gamma R_{2} .
$$

Define $\Omega_{2}:=\left\{y \in \mathscr{B}:\|y\|<R_{2}\right\}$; using (4.3) and (4.15) for $s \in\left[\xi_{1}, \xi_{2}\right]$, we get

$$
\begin{aligned}
(T y)(\tau) & =\lambda\left(\int_{t_{1}}^{t_{n}} G(\tau, s) h(s) f(y(s)) \Delta s+A(h f(y)) \psi(\tau)+B(h f(y)) \phi(\tau)\right) \\
& \geq \lambda \int_{\xi_{1}}^{\xi_{2}} G(\tau, s) h(s) f(y(s)) \Delta s \geq \lambda\left(f_{\infty}-\epsilon\right) \int_{\xi_{1}}^{\xi_{2}} G(\tau, s) h(s) y(s) \Delta s \\
& \geq \lambda\left(f_{\infty}-\epsilon\right) \Gamma R_{2} \int_{\xi_{1}}^{\xi_{2}} G(\tau, s) h(s) \Delta s \geq R_{2}=\|y\|,
\end{aligned}
$$

where we have used the left-hand side of (4.9). Hence we have shown that

$$
\|T y\| \geq\|y\|, \quad y \in \mathscr{P} \cap \partial \Omega_{2}
$$

An application of Theorem 4.1 yields the conclusion of the theorem; in other words, $T$ has a fixed point $y$ in $\mathscr{P} \cap\left(\bar{\Omega}_{2} \backslash \Omega_{1}\right)$ with $R_{1} \leq\|y\| \leq R_{2}$. 
Theorem 4.3. Suppose (1.3), (1.4), (3.25), (4.1), and (4.3) hold. Then for each $\lambda$ satisfying

$$
\frac{1}{f_{0} \Gamma \int_{\xi_{1}}^{\xi_{2}} G(\tau, s) h(s) \Delta s}<\lambda<\frac{1}{f_{\infty} K}
$$

there exists at least one positive solution of (1.1), (1.2) in $\mathscr{P}$.

Proof. Let $\lambda$ be as in (4.18) and let $\eta>0$ be such that

$$
\frac{1}{\left(f_{0}-\eta\right) \Gamma \int_{\xi_{1}}^{\xi_{2}} G(\tau, s) h(s) \Delta s} \leq \lambda \leq \frac{1}{\left(f_{\infty}+\eta\right) K}
$$

Again let $T$ be the operator defined in (4.7). We once more seek a fixed point of $T$ in $\mathscr{P}$ by establishing the hypotheses of Theorem 4.1.

First, consider $f_{0}$. There exists an $R_{1}>0$ such that $f(y) \geq\left(f_{0}-\eta\right) y$ for $0<y \leq R_{1}$ by the definition of $f_{0}$. Pick $y \in \mathscr{P}$ with $\|y\|=R_{1}$. For $s \in\left[\xi_{1}, \xi_{2}\right]$, where $\xi_{1}, \xi_{2}$ are as in Lemma 3.5, we have

$$
y(s) \geq \Gamma\|y\|=\Gamma R_{1} .
$$

Using the left-hand side of (4.19) and (4.20) we get, for $s \in\left[\xi_{1}, \xi_{2}\right]$,

$$
\begin{aligned}
(T y)(\tau) & =\lambda\left(\int_{t_{1}}^{t_{n}} G(\tau, s) h(s) f(y(s)) \Delta s+A(h f(y)) \psi(\tau)+B(h f(y)) \phi(\tau)\right) \\
& \geq \lambda\left(f_{0}-\eta\right) \int_{\xi_{1}}^{\xi_{2}} G(\tau, s) h(s) y(s) \Delta s \geq \lambda\left(f_{0}-\eta\right) R_{1} \Gamma \int_{\xi_{1}}^{\xi_{2}} G(\tau, s) h(s) \Delta s \\
& \geq R_{1}=\|y\| .
\end{aligned}
$$

Therefore $\|T y\| \geq\|y\|$. This motivates us to define

$$
\Omega_{1}:=\left\{y \in \mathscr{B}:\|y\|<R_{1}\right\}
$$

whereby our work above confirms

$$
\|T y\| \geq\|y\|, \quad y \in \mathscr{P} \cap \partial \Omega_{1} .
$$

Next consider $f_{\infty}$. Again by definition there exists an $R_{2}^{\prime}>R_{1}$ such that $f(y) \leq\left(f_{\infty}+\eta\right) y$ for $y \geq R_{2}^{\prime}$. If $f$ is bounded, there exists $M>0$ with $f(y) \leq M$ for all $y \in(0, \infty)$. Let

$$
R_{2}:=\max \left\{2 R_{2}^{\prime}, \lambda M\left(\int_{t_{1}}^{t_{n}} G(s, s) h(s) \Delta s+A(h) \psi\left(t_{n}\right)+B(h) \phi\left(\rho\left(t_{1}\right)\right)\right)\right\}
$$


12 Second-order $n$-point eigenvalue problems on time scales

If $y \in \mathscr{P}$ with $\|y\|=R_{2}$, then we have

$$
\begin{aligned}
(T y)(t) & \leq \lambda\left(\int_{t_{1}}^{t_{n}} G(s, s) h(s) f(y(s)) \Delta s+A(h f(y)) \psi\left(t_{n}\right)+B(h f(y)) \phi\left(\rho\left(t_{1}\right)\right)\right) \\
& \leq \lambda M\left(\int_{t_{1}}^{t_{n}} G(s, s) h(s) \Delta s+A(h) \psi\left(t_{n}\right)+B(h) \phi\left(\rho\left(t_{1}\right)\right)\right) \leq R_{2}=\|y\| .
\end{aligned}
$$

As a result, $\|T y\| \leq\|y\|$. Thus, take

$$
\Omega_{2}:=\left\{y \in \mathscr{B}:\|y\|<R_{2}\right\}
$$

so that $\|T y\| \leq\|y\|$ for $y \in \mathscr{P} \cap \partial \Omega_{2}$. If $f$ is unbounded, take $R_{2}:=\max \left\{2 R_{1}, R_{2}^{\prime}\right\}$ such that $f(y) \leq f\left(R_{2}\right)$ for $0<y \leq R_{2}$. If $y \in \mathscr{P}$ with $\|y\|=R_{2}$, then we have

$$
\begin{aligned}
(T y)(t) & \leq \lambda f\left(R_{2}\right)\left(\int_{t_{1}}^{t_{n}} G(s, s) h(s) \Delta s+A(h) \psi\left(t_{n}\right)+B(h) \phi\left(\rho\left(t_{1}\right)\right)\right) \\
& \leq \lambda\left(f_{\infty}+\eta\right) R_{2} K \leq R_{2}=\|y\|,
\end{aligned}
$$

where we have used the left-hand side of (4.19). Hence we have shown that

$$
\|T y\| \leq\|y\|, \quad y \in \mathscr{P} \cap \partial \Omega_{2}
$$

if we take

$$
\Omega_{2}:=\left\{y \in \mathscr{B}:\|y\|<R_{2}\right\}
$$

As before, an application of Theorem 4.1 yields the conclusion that $T$ has a fixed point $y$ in $\mathscr{P} \cap\left(\bar{\Omega}_{2} \backslash \Omega_{1}\right)$ with $R_{1} \leq\|y\| \leq R_{2}$.

Corollary 4.4. Suppose (1.3), (1.4), (3.25), and (4.1) hold. If $f$ is sublinear (i.e., $f_{0}=\infty$ and $f_{\infty}=0$ ), or if $f$ is superlinear (i.e., $f_{0}=0$ and $f_{\infty}=\infty$ ), then for any $\lambda>0$ the boundary value problem (1.1)-(1.2) has at least one positive solution in $\mathscr{P}$.

Proof. For the superlinear claim, use (4.8) of Theorem 4.2; for the sublinear claim, use (4.18) of Theorem 4.3.

\section{Examples}

Example 5.1. Let $\mathbb{T}=\mathbb{R}$, and consider the three-point boundary value problem

$$
\begin{gathered}
y^{\prime \prime}-y+\lambda f(y)=0, \quad-1<t<1, \\
y(-1)=a y(0)=y(1),
\end{gathered}
$$

where $a:=\sinh (2) / 4 \sinh (1)$ and $f \in C([0, \infty),[0, \infty))$ such that $f_{0}$ and $f_{\infty}$ exist. 
It is easy to check that

$$
\begin{gathered}
\psi(t)=\frac{e^{t+1}-e^{-t-1}}{2}=\sinh (1+t), \quad \phi(t)=\frac{e^{1-t}-e^{t-1}}{2}=\sinh (1-t), \\
d=\left|\begin{array}{cc}
\phi(1) & \psi(1) \\
\phi^{\prime}(1) & \psi^{\prime}(1)
\end{array}\right|=\sinh (2) .
\end{gathered}
$$

Since

$$
\begin{gathered}
D=\left|\begin{array}{cc}
-a \psi(0) & d-a \phi(0) \\
d-a \psi(0) & -a \phi(0)
\end{array}\right|=-\frac{1}{2} \sinh ^{2}(2)<0, \\
d-a \phi(0)=d-a \psi(0)=\frac{3}{4} \sinh (2)>0,
\end{gathered}
$$

(3.25) holds. We take $\left[\xi_{1}, \xi_{2}\right]=[-1 / 2,1 / 2]$, so that

$$
\begin{gathered}
\Gamma=\min \left\{\frac{\phi(1 / 2)}{\phi(-1)}, \frac{\psi(-1 / 2)}{\psi(1)}\right\}=\frac{\sinh (1 / 2)}{\sinh (2)}, \\
A(1)=\frac{1}{D}\left|\begin{array}{ll}
a \int_{-1}^{1} G(0, s) d s & d-a \phi(0) \\
a \int_{-1}^{1} G(0, s) d s \quad-a \phi(0)
\end{array}\right|=\frac{(e-1)^{2}}{2 e \sinh (2)}, \\
B(1)=\frac{1}{D}\left|\begin{array}{cc}
-a \psi(0) & a \int_{-1}^{1} G(0, s) d s \\
d-a \psi(0) & a \int_{-1}^{1} G(0, s) d s
\end{array}\right|=\frac{(e-1)^{2}}{2 e \sinh (2)}, \\
K=\frac{1}{d} \int_{-1}^{1} \psi(s) \phi(s) d s+A(1) \psi(1)+B(1) \phi(-1)=\frac{\cosh (2)}{\sinh (2)}+e+\frac{1}{e}-\frac{5}{2} .
\end{gathered}
$$

Note that $\tau$ in (4.3) is determined by

$$
\begin{gathered}
\max \left\{t \in\left[-1,-\frac{1}{2}\right]: \frac{\psi(t)}{d} \int_{-1 / 2}^{1 / 2} \phi(s) d s, t \in\left[\frac{1}{2}, 1\right]: \frac{\phi(t)}{d} \int_{-1 / 2}^{1 / 2} \psi(s) d s,\right. \\
\left.t \in\left(-\frac{1}{2}, \frac{1}{2}\right): \frac{\phi(t)}{d} \int_{-1 / 2}^{t} \psi(s) d s+\frac{\psi(t)}{d} \int_{t}^{1 / 2} \phi(s) d s\right\}
\end{gathered}
$$

which is

$$
\frac{\phi(0)}{d} \int_{-1 / 2}^{0} \psi(s) d s+\frac{\psi(0)}{d} \int_{0}^{1 / 2} \phi(s) d s=2 \frac{\sinh (1)}{\sinh (2)}\left(\cosh (1)-\cosh \left(\frac{1}{2}\right)\right) .
$$


14 Second-order $n$-point eigenvalue problems on time scales

Applying (5.4) and (5.6), we can find the interval in (4.8):

$$
\frac{\sinh ^{2}(2)}{2 \sinh (1) \sinh (1 / 2)(\cosh (1)-\cosh (1 / 2)) f_{\infty}}<\lambda<\frac{1}{K f_{0}},
$$

approximately

$$
\frac{25.8511}{f_{\infty}}<\lambda<\frac{0.615962}{f_{0}}
$$

Example 5.2. Let $\mathbb{T}=h \mathbb{Z}$ for $h=2^{-10}$, and consider the four-point boundary value problem

$$
\begin{gathered}
\left(p y^{\nabla}\right)^{\Delta}(t)+\lambda f(y)=0, \quad 0<t<1, \\
y(0)-p(0) y^{\nabla}(0)=\frac{2}{5}\left(y\left(\frac{1}{4}\right)+y\left(\frac{3}{4}\right)\right), \\
y(1)+p(1) y^{\nabla}(1)=\frac{2}{5}\left(y\left(\frac{1}{4}\right)+y\left(\frac{3}{4}\right)\right),
\end{gathered}
$$

where $p(t):=1 /(t+h)(t+2 h)$ and $f \in C([0, \infty),[0, \infty))$ such that $f_{0}$ and $f_{\infty}$ exist.

Then direct calculation verifies that

$$
\begin{gathered}
\psi(t)=\frac{1}{3}(t+h)(t+2 h)(t+3 h)+1-2 h^{3}, \\
\phi(t)=\frac{1}{3}(1+h)(1+2 h)(1+3 h)+1-\frac{1}{3}(t+h)(t+2 h)(t+3 h), \\
d=\psi(1)+p(1) \frac{(\psi(1)-\psi(1-h))}{h}=\frac{1}{3}\left(11 h^{2}+6 h+7\right), \\
D=\left|\begin{array}{cc}
-\frac{2}{5}\left(\psi\left(\frac{1}{4}\right)+\psi\left(\frac{3}{4}\right)\right) & d-\frac{2}{5}\left(\phi\left(\frac{1}{4}\right)+\phi\left(\frac{3}{4}\right)\right) \\
d-\frac{2}{5}\left(\psi\left(\frac{1}{4}\right)+\psi\left(\frac{3}{4}\right)\right) & -\frac{2}{5}\left(\phi\left(\frac{1}{4}\right)+\phi\left(\frac{3}{4}\right)\right)
\end{array}\right|=\frac{-d^{2}}{5} .
\end{gathered}
$$

Moreover, since

$$
\begin{aligned}
& d-\frac{2}{5}\left(\psi\left(\frac{1}{4}\right)+\psi\left(\frac{3}{4}\right)\right)=\frac{1}{40}\left(59+60 h+88 h^{2}\right)>0, \\
& d-\frac{2}{5}\left(\phi\left(\frac{1}{4}\right)+\phi\left(\frac{3}{4}\right)\right)=\frac{1}{40}\left(53+36 h+88 h^{2}\right)>0,
\end{aligned}
$$


(3.25) holds. Let $\left[\xi_{1}, \xi_{2}\right]=[0,1 / 2]$, so that

$$
\begin{gathered}
\Gamma=\min \left\{\frac{\phi(1 / 2)}{\phi(-h)}, \frac{\psi(0)}{\psi(1)}\right\}=\frac{\psi(0)}{\psi(1)}=\frac{3}{11 h^{2}+6 h+4}, \\
A(1)=\frac{1}{D}\left|\begin{array}{l}
\frac{2}{5} \sum_{s=0}^{1 / h-1} G\left(\frac{1}{4}, s h\right) h+\frac{2}{5} \sum_{s=0}^{1 / h-1} G\left(\frac{3}{4}, s h\right) h \quad d-\frac{2}{5}\left(\phi\left(\frac{1}{4}\right)+\phi\left(\frac{3}{4}\right)\right) \\
\frac{2}{5} \sum_{s=0}^{1 / h-1} G\left(\frac{1}{4}, s h\right) h+\frac{2}{5} \sum_{s=0}^{1 / h-1} G\left(\frac{3}{4}, s h\right) h-\frac{2}{5}\left(\phi\left(\frac{1}{4}\right)+\phi\left(\frac{3}{4}\right)\right)
\end{array}\right|, \\
B(1)=\frac{1}{D}\left|\begin{array}{l}
-\frac{2}{5}\left(\psi\left(\frac{1}{4}\right)+\psi\left(\frac{3}{4}\right)\right) \\
\left.d-\frac{2}{5}\left(\psi\left(\frac{1}{4}\right)+\psi\left(\frac{3}{4}\right)\right) \frac{2}{5} \sum_{s=0}^{1 / h-1} G\left(\frac{1}{4}, s h\right) h+\frac{2}{5} \sum_{s=0}^{1 / h-1} G\left(\frac{3}{4}, s h\right) h\right) h+\frac{2}{5} \sum_{s=0}^{1 / h-1} G\left(\frac{3}{4}, s h\right) h
\end{array}\right|, \\
K=\frac{1}{d} \sum_{s=0}^{1 / h-1} \psi(s h) \phi(s h) h+A(1) \psi(1)+B(1) \phi(-h) \approx 3.02392 .
\end{gathered}
$$

As in the previous example, we determine $\tau$ in (4.3) from

$$
\begin{gathered}
\max \left\{t \in[-h, 0]: \frac{\psi(t) h}{d} \sum_{s=0}^{(1 / 2 h)-1} \phi(s h), t \in\left[\frac{1}{2}, 1\right]: \frac{\phi(t) h}{d} \sum_{s=0}^{(1 / 2 h)-1} \psi(s h),\right. \\
\left.t \in\left(0, \frac{1}{2}\right): \frac{\phi(t) h}{d} \sum_{s=0}^{t / h-1} \psi(s h)+\frac{\psi(t) h}{d} \sum_{s=t / h}^{(1 / 2 h)-1} \phi(s h)\right\}
\end{gathered}
$$

which is

$$
\frac{\phi(290 h) h}{d} \sum_{s=0}^{289} \psi(s h)+\frac{\psi(290 h) h}{d} \sum_{s=290}^{(1 / 2 h)-1} \phi(s h) \approx 0.284188
$$

Applying (5.14) and (5.15), we can find an approximate interval for (4.8):

$$
\frac{4.69862}{f_{\infty}}<\lambda<\frac{0.330697}{f_{0}}
$$

\section{Acknowledgment}

The second author is partly supported by NSFC (no. 10271095). 


\section{References}

[1] D. R. Anderson, Extension of a second-order multi-point problem to time scales, Dynamic Systems and Applications 12 (2003), no. 3-4, 393-403.

[2] _ Nonlinear triple-point problems on time scales, Electronic Journal of Differential Equations 2004 (2004), no. 47, 1-12.

[3] _ , Twin n-point boundary value problems, Applied Mathematics Letters 17 (2004), no. 9, 1053-1059.

[4] D. R. Anderson and R. I. Avery, An even-order three-point boundary value problem on time scales, Journal of Mathematical Analysis and Applications 291 (2004), no. 2, 514-525.

[5] D. R. Anderson, R. I. Avery, and J. Henderson, Existence of solutions for a one dimensional pLaplacian on time-scales, Journal of Difference Equations and Applications 10 (2004), no. 10, 889-896.

[6] F. M. Atici and G. Sh. Guseinov, On Green's functions and positive solutions for boundary value problems on time scales, Journal of Computational and Applied Mathematics 141 (2002), no. 1-2, 75-99.

[7] B. Aulbach and S. Hilger, Linear dynamic processes with inhomogeneous time scale, Nonlinear Dynamics and Quantum Dynamical Systems (Gaussig, 1990), Math. Res., vol. 59, Akademie, Berlin, 1990, pp. 9-20.

[8] M. Bohner and H. Luo, Singular second-order multipoint dynamic boundary value problems with mixed derivatives, to appear in Advances in Difference Equations.

[9] M. Bohner and A. Peterson, Dynamic Equations on Time Scales. An Introduction with Applications, Birkhäuser Boston, Massachusetts, 2001.

[10] M. Bohner and A. Peterson (eds.), Advances in Dynamic Equations on Time Scales, Birkhäuser Boston, Massachusetts, 2003.

[11] C. J. Chyan and J. Henderson, Eigenvalue problems for nonlinear differential equations on a measure chain, Journal of Mathematical Analysis and Applications 245 (2000), no. 2, 547-559.

[12] J. J. DaCunha, J. M. Davis, and P. K. Singh, Existence results for singular three point boundary value problems on time scales, Journal of Mathematical Analysis and Applications 295 (2004), no. 2, 378-391.

[13] J. M. Davis, J. Henderson, K. Rajendra Prasad, and W. Yin, Solvability of a nonlinear second order conjugate eigenvalue problem on a time scale, Abstract and Applied Analysis 5 (2000), no. 2, 9199.

[14] S. Hilger, Analysis on measure chains - a unified approach to continuous and discrete calculus, Results in Mathematics 18 (1990), no. 1-2, 18-56.

[15] E. R. Kaufmann, Positive solutions of a three-point boundary-value problem on a time scale, Electronic Journal of Differential Equations 2003 (2003), no. 82, 1-11.

[16] E. R. Kaufmann and Y. N. Raffoul, Eigenvalue problems for a three-point boundary-value problem on a time scale, Electronic Journal of Qualitative Theory of Differential Equations (2004), no. 2, $1-10$.

[17] L. Kong and Q. Kong, Positive solutions of nonlinear m-point boundary value problems on a measure chain, Journal of Difference Equations and Applications 9 (2003), no. 1, 121-133.

[18] M. A. Krasnosel'skiĭ, Positive Solutions of Operator Equations, P. Noordhoff, Groningen, 1964.

[19] R. Ma and B. Thompson, Positive solutions for nonlinear m-point eigenvalue problems, Journal of Mathematical Analysis and Applications 297 (2004), no. 1, 24-37.

[20] A. C. Peterson, Y. N. Raffoul, and C. C. Tisdell, Three point boundary value problems on time scales, Journal of Difference Equations and Applications 10 (2004), no. 9, 843-849. 
[21] H. R. Sun and W.-T. Li, Positive solutions for nonlinear three-point boundary value problems on time scales, Journal of Mathematical Analysis and Applications 299 (2004), no. 2, 508-524.

[22] H. R. Sun and W. T. Li, Positive solutions for nonlinear m-point boundary value problems on time scales, Acta Mathematica Sinica 49 (2006), no. 2, 369-380.

Douglas R. Anderson: Department of Mathematics and Computer Science, Concordia College, Moorhead, MN 56562, USA

E-mail address: andersod@cord.edu

Ruyun Ma: Department of Mathematics, Northwest Normal University, Lanzhou 730070, China E-mail address: mary@nwnu.edu.cn 